\title{
Neural Networks in Business Forecasting
}

\author{
Dr. Osman Mohamed Abbas*
}

Email: Osman.sd@live.com

\begin{abstract}
Neural Network is defined as the ability of a group to solve more problems than its individual members. The idea brings that a group of people can solve problems efficiently and offer greater insight and a better answer than any one individual could provide. The applications of Neural Network enhance an innovative business model for an enterprise. Role of Neural Network in an enterprise brings effectiveness. Further work will be carried out towards the Mathematical modeling of neural networks and various parameters will be engaged so as to get the required result to desired degree of accuracy [1].
\end{abstract}

Keywords: Forecasting, Neural Networks, Business

\section{Introduction}

Forecasting has been dominated by linear methods for many decades. Linear methods are easy to develop and implement and they are also relatively simple to understand and interpret. However, linear models have serious limitation in that they are not able to capture any nonlinear relationships in the data [2]. The approximation of linear models to complicated nonlinear relationships is not always satisfactory. In the early 1980s, Makridakis (1982) organized a large-scale forecasting competition (often called M-competition) where a majority of commonly used linear methods were tested with more than 1,000 real time series data. The mixed results show that no single linear model is globally the best, which may be interpreted as the failure of linear modeling in accounting for a varying degree of nonlinearity that is common in real world problems [3]. Artificial neural networks are mathematical models inspired by the organization and functioning of biological neurons. There are numerous artificial neural network variations that are related to the nature of the task assigned to the network [4]. There are also numerous variations in how the neuron is modeled. In some cases, these models correspond closely to biological neurons and in other cases the models depart from biological functioning in significant ways. See the appendix for a more detailed explanation of the artificial neural network paradigm [5].

* Corresponding author. 
In the human brain, a typical neuron collects signal from others through a host of fine structures called dendrites. The neuron sends out spikes of electrical activity through a long thin strand known as an axon, which splits into thousands of branches. At the end of each branch, a structure called a synapse converts the activity from the axon into electrical effects that inhibit or excite activity in the connected neurons [6]. When a neuron receives excitatory input that is sufficiently large compared with its inhibitory input, it sends a spike of electrical activity down its axiom. Learning occurs by changing the effectiveness of the synapses so that the influence of one neuron on another changes. An artificial neural network is a simulation of biological brain. The purpose of a neural network is to learn to recognize pattern in our data. Once the neural network has been trained on sample of our data it can make predictions by detecting similar patterns in future data. Thus a neural network is a computational system inspired by the structure, processing method, learning ability of a biological brain [7].

Market Forecast

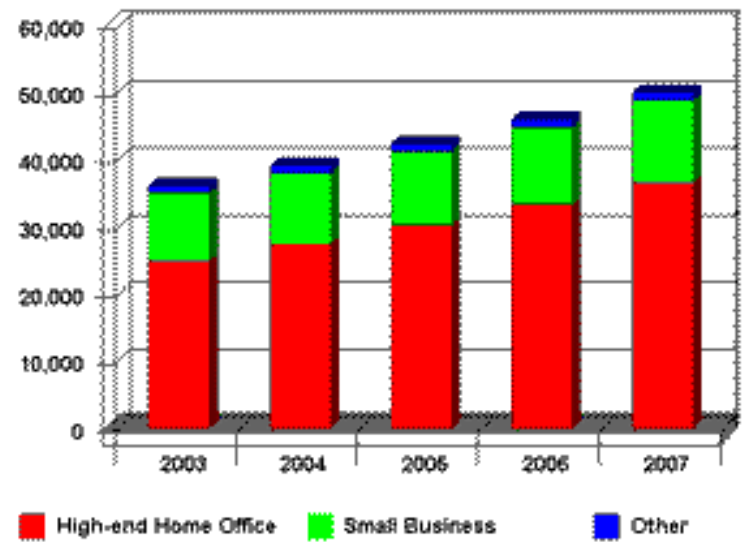

Figure 1: Market Forecasting

\section{Forecasting}

Forecasting is the process of making predictions of the future based on past and present data and analysis of trends. A commonplace example might be estimation of some variable of interest at some specified future date. Prediction is a similar, but more general term. Both might refer to formal statistical methods employing time series, cross-sectional or longitudinal data, or alternatively to less formal judgmental methods [8]. Usage can differ between areas of application: for example, in hydrology, the terms "forecast" and "forecasting" are sometimes reserved for estimates of values at certain specific future times, while the term "prediction" is used for more general estimates, such as the number of times floods will occur over a long period. Risk and uncertainty are central to forecasting and prediction; it is generally considered good practice to indicate the degree of uncertainty attaching to forecasts. In any case, the data must be up to date in order for the forecast to be as accurate as possible [9].

Forecasts are constantly made in business, finance, economics, government, and many other fields, and they guide many important decisions. As with anything else, there are good and bad ways to forecast. This book is about the good ways: modern, rigorous, replicable, largely-quantitative statistical/econometric methods - their 
strengths and their limitations. That's why I dislike the above picture of the crystal ball - it bows to the common misconception among the uninitiated that forecasting is some sort of dubious mystical activity, like fortune telling or astrology. But how could a forecasting book not begin with a picture like that? So I decided to lighten up, if only for a moment [10]. Forecasts are made and used in numerous fields. To develop a feel for the tremendous diversity of forecasting applications, let's sketch some of the areas where forecasts feature prominently, and the corresponding diversity of decisions that they support [11]. One key field is economics, broadly defined. Governments, businesses, policy organizations, central banks, financial services firms, and economic consulting firms around the world routinely forecast the major economic variables, such as gross domestic product (GDP), unemployment, consumption, investment, the price level, and interest rates. Governments use such forecasts to guide monetary and fiscal policy, and private firms use them for strategic planning, because economy-wide economic fluctuations typically have industry-level and firm-level effects. In addition to forecasting "standard" variables such as GDP, economists sometimes make more exotic forecasts, such as the stage of the business cycle that we'll be in six months from now (expansion or contraction), the state of future stock market activity (bull or bear), or the state of future foreign exchange market activity (appreciation or depreciation) [12]. Again, such forecasts are of obvious use to both governments and firms - if they're accurate! Another key area is business and all its subfields. These include management strategy of all types including operations management and control (hiring, production, inventory, investment), marketing (pricing distributing, advertising,), and accounting (budgeting using revenue and expenditure forecasts), etc. Sales forecasting is a good example. Firms routinely forecast sales to help guide management decisions in inventory management, sales force management, and production planning, as well as strategic planning regarding product lines, new market entry, and so on. More generally, firms use forecasts to decide what to produce (What product or mix of products should be produced?), when to produce (Should we build up inventories now in anticipation of high future demand? How many shifts should be run?), how much to produce and how much capacity to build (What are the trends in market size and market share? Are there cyclical or seasonal effects? How quickly and with what pattern will a newly-built plant or a newly-installed technology depreciate?), and where to produce (Should we have one plant or many? If many, where should we locate them?). Firms also use forecasts of future prices and availability of inputs to guide production decisions [13].

Forecasts are always wrong, but some are "more wrong" than others. Forecasting the demand for innovative products, fashion goods, and the like is generally more difficult than forecasting demand for more "commoditylike” products that are sold on a daily basis. Aggregate forecasts of a group of similar products are generally more accurate than individual forecasts of the individual products that make up the group [14]. Finally, the longer the forecast into the future, the less reliable the forecast will be. Forecasting practice is based on a mix of qualitative and quantitative methods. When planning occurs for innovative products, little demand data are available for the product of interest and the degree to which like product demand data are similar is unknown. Thus a large amount of judgment is needed by experts who can use their industry expertise to predict demand [15]. These experts, though, will undoubtedly use historical demand data, even if not directly, in their judgment. Commodity-like products that are sold every day, on the other hand, are much more suitable for quantitative models and need very little judgment to forecast demand. Still, when knowledge of certain events leads one to believe that future demand might not track historical trends, some judgment may be warranted to make 
adjustments in the models which use past data [16]. In this case, a heavy reliance on past data with adjustments based on expert judgment should be the method used for forecasting. Forecasting should be done primarily for end-item demand. In manufacturing situations, this means there is no real need for forecasting component parts which make up the final item. When production quantities for the end item have been determined, component demand can be computed based on the production plan of the end item and knowledge of the bill of materials (BOM) [17].

Aggregating forecasts across multiple items reduces forecasting errors. A clothing store, for instance, might be able to estimate within a pretty narrow range what the demand will be for men's dress shirts. But when that store tries to estimate the demand for individual styles, colors, and sizes of shirts, the accuracy of their forecasts will be considerably worse. Firms handle this kind of forecasting problem usually in one of three ways; they either forecast from the bottom up, from the top down, or they start in the middle and work both up and down [18]. The "top down" forecast essentially estimates total sales demand and then divides those sales dollars level by level until the stock keeping unit (SKU) is reached. The "bottom up" method, as one might expect, starts with forecasts at the SKU level and then aggregates those demand estimates level by level to reach a companylevel forecast. Another method, one might call the "in-between" method, starts forecasts at the category level (like men's dress shirts), and then works up to determine store sales and works down to divide up the forecast into styles, colors and SKUs [19].

The use of management time to make forecasts is relatively expensive when compared to the cost of using statistical forecasting models, and the difference between the costs of these two methods has been increasing in recent years due to the automated acquisition of data from point of sale systems and computer power in general [20]. There can be no substitute for human input in the forecasting process; however, human input can be expensive. In addition, research indicates that for some everyday commodity-type items, simple statistical models work well and in fact work better when not massaged by managers. Still, some managers believe that spending time to make forecasts perfect will solve most of their supply chain problems. There are times when managerial input is needed, but there comes a point where it is better to understand the inaccuracy in the forecast and plan accordingly. Once a good forecasting process (procedures, techniques, models and management oversight) has been put in place, continual refinement has little value and can even hurt the forecasting process [21].

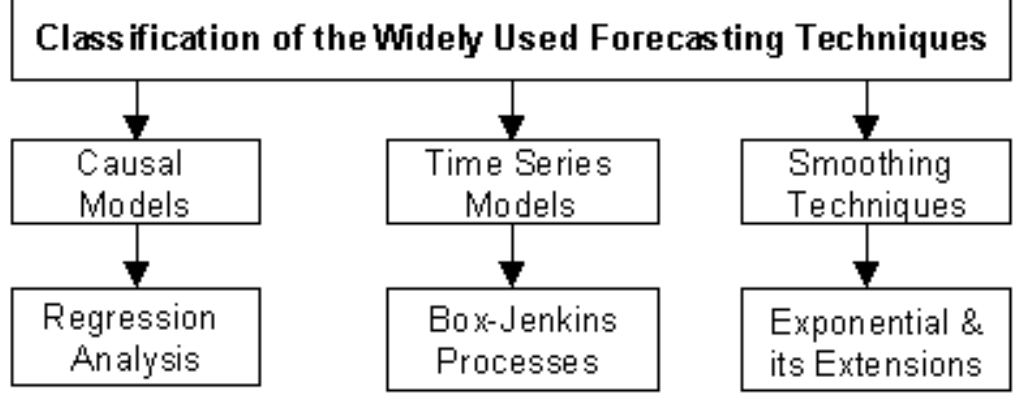

Figure 2: Forecasting Model 


\section{Neural networks}

Artificial neural networks (ANNs) are computing models for information processing and pattern identification. They grow out of research interest in modeling biological neural systems, especially human brains. An ANN is a network of many simple computing units called neurons or cells, which are highly interconnected and organized in layers. Each neuron performs the simple task of information processing by converting received inputs into processed outputs [22]. Through the linking arcs among these neurons, knowledge can be generated and stored regarding the strength of the relationship between different nodes. Although the ANN models used in all applications are much simpler than actual neural systems, they are able to perform a variety of tasks and achieve remarkable results [23]. Over the last several decades, many types of ANN models have been developed, each aimed at solving different problems. But by far the most widely and successfully used for forecasting has been the feed forward type neural network. Figure 1 shows the architecture of a three-layer feed forward neural network that consists of neurons (circles) organized in three layers: input layer, hidden layer, and output layer. The neurons in the input nodes correspond to the independent or predictor variables ( $\mathrm{x}$ ) that are believed to be useful for forecasting the dependent variable (y) which corresponds to the output neuron. Neurons in the hidden layer are connected to both input and output neurons and are key to learning the pattern in the data and mapping the relationship from input variables to the output variable. With nonlinear transfer functions, hidden neurons can process complex information received from input neurons and then send processed information to the output layer for further processing to generate forecasts. In feed forward ANNs, the information flow is one directional from the input layer to the hidden layer then to the output layer without any feedback from the output layer [24].

Artificial neural networks (ANN) have been widely touted as solving many forecasting and decision modeling problems . For example, they are argued to be able to model easily any type of parametric or non-parametric process and automatically and optimally transform the input data. These sorts of claims have led to much interest in artificial neural networks [25]. On the other hand, Chatfield (1993) has queried whether artificial neural networks have been oversold or are just a fad. In this paper, we will attempt to give a balanced review of the literature comparing artificial neural networks and statistical techniques. Our review will be segmented into three different application areas: time series forecasting, regression based forecasting, and regression-based decision models. Additionally, we will note the literature comparing artificial neural networks and other models such as discriminant analysis. But before that review, we will first examine the general claims made for artificial neural networks that are relevant to forecasting and decision making [26].

\section{Neural network as a forecasting tool}

A neural network is a collection of interconnected simple processing elements. Every connection in a neural network has a weight attached to it. There are countless learning methods for neural networks. However, they can be classified into two groups, namely supervised and unsupervised method. Supervised learning requires historical data with examples of both dependent and independent variables to train the network. The known answers are worked as a teacher to correct the behavior of the training network. Hopfield, Boltzman, Adaline, Back propagation, are some of the well- known supervised learning methods. It is commonly used to build prediction, classification and time series models. Unsupervised learning method creates its own model to 
interpret the data without known answers. Adaptive resonance theory, Kohonen self- organizing map counter propagation network are some of the popularly used unsupervised learning approaches. They are often used for clustering data. The back propagation algorithm has emerged as one of the most widely used learning procedures for multilayer networks [27]. A typical back propagation neural network usually has an input layer, some hidden layers and an output layer. The units in the network are connected in a feed forward manner, from the input layer to the output layer. The weights of connections have been given initial values. The error between the predicted output value and the actual value is back propagated through the network for the updating of the weights. This is a supervised learning procedure that attempts to minimize the error between the desired and the predicted outputs [28].

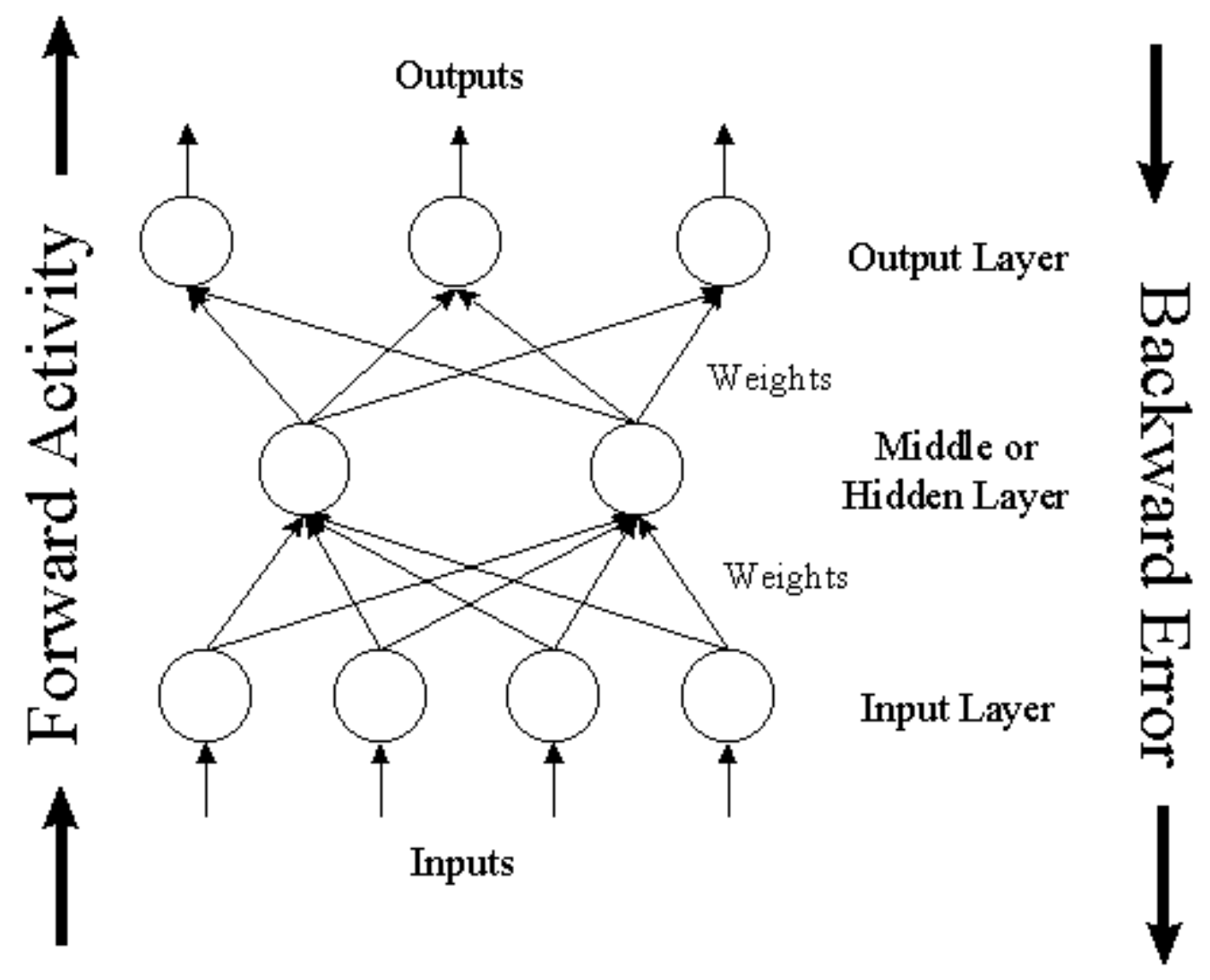

Figure 3: Neural Network Model

There is a marketing application which has been integrated with a neural network system. The Airline Marketing Tactician (a trademark abbreviated as AMT) is a computer system made of various intelligent technologies including expert systems. A feed forward neural network is integrated with the AMT and was trained using back propagation to assist the marketing control of airline seat allocations. The adaptive neural approach was amenable to rule expression. Additionally, the application's environment changed rapidly and constantly, which required a continuously adaptive solution. The system is used to monitor and recommend booking advice for each departure. Such information has a direct impact on the profitability of an airline and can provide a technological advantage for users of the system [29]. 
While it is significant that neural networks have been applied to this problem, it is also important to see that this intelligent technology can be integrated with expert systems and other approaches to make a functional system. Neural networks were used to discover the influence of undefined interactions by the various variables. While these interactions were not defined, they were used by the neural system to develop useful conclusions. It is also noteworthy to see that neural networks can influence the bottom line [30].

Neural networks change the way to use information in marketing. With such a new information technology, a company using a neural network, will eventually have affordable, near real-time access to all the raw numbers it wants. These data may be obtained from consumer credit card applications, point-of -purchase credit-card sales, and credit agency reports. The real difference among competitors will be the quality of analysis each performs and the capacity of decisions flowing from it. Neural networks help managers gather and process information, such as age, income, credit history, and products purchased [31].

Neural networks have been applied to a wide range of information-processing activities, such as associate memory, pattern classification and clustering, and function approximation and prediction. These applications are characterized by unstructured decision processes, multi objectives and multiple stage decision activities. Such applications may not be efficiently supported using expert and decision support systems technologies [32].

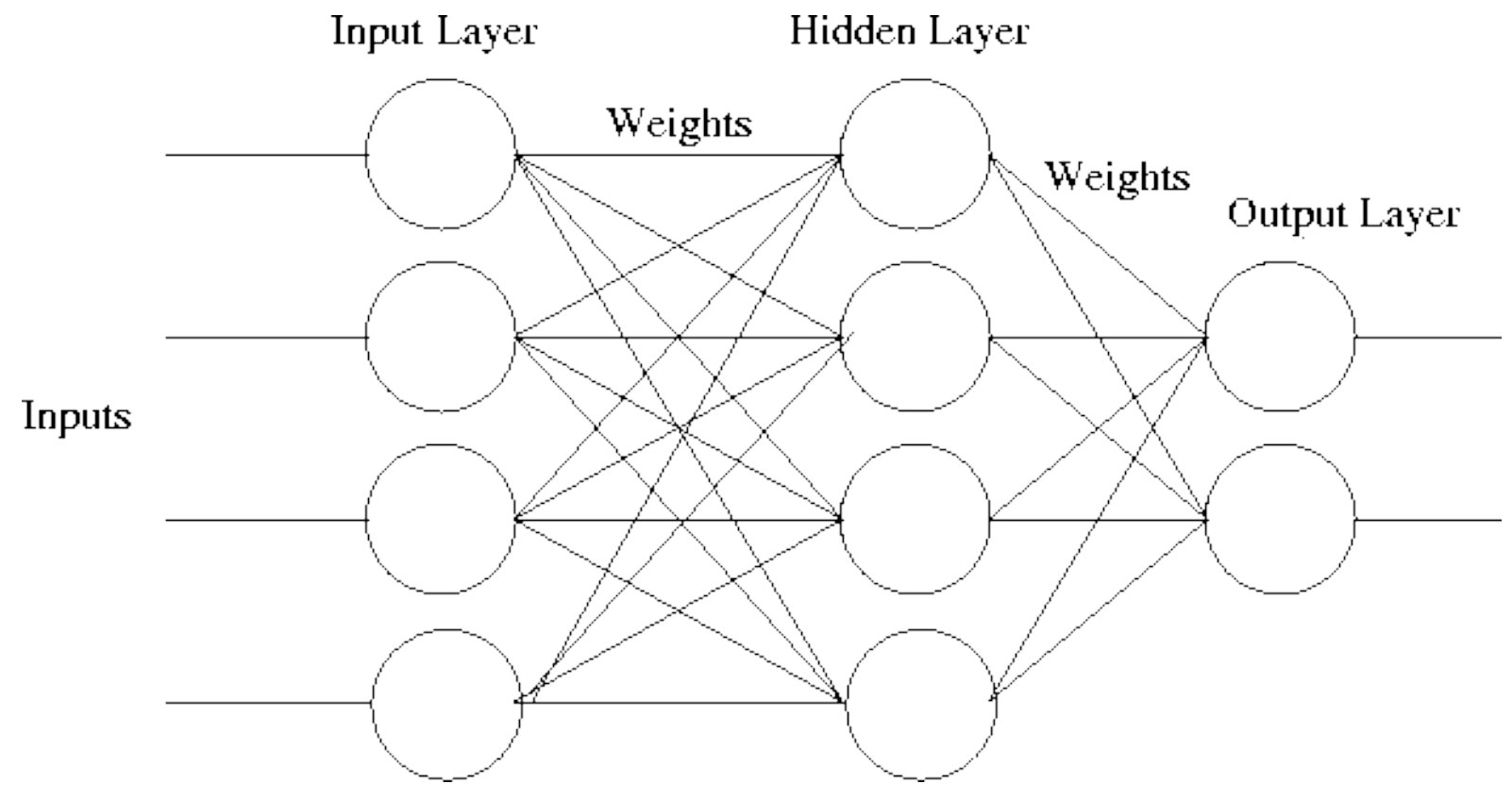

Figure 4: Multilayer Neural Network

\section{Overview of business applications}

Over the last decade, neural networks have found application across a wide range of areas from business, commerce and industry. In this section, an overview is provided of the kinds of business problems to which neural networks are suited, with a brief discussion of some of the reported studies relevant to each area. This 
overview is based upon some excellent review articles [3,48,49], as well as many published studies [33].

The goal of modern marketing exercises is to identify customers who are likely to respond positively to a product, and to target any advertising or solicitation towards these customers. Target marketing involves market segmentation, where by the market is divided into distinct groups of customers with very different consumer behavior. Market segmentation can be achieved using neural networks by segmenting customers according to basic characteristics including demographics, socio-economic status, geographic location, purchase patterns, and attitude towards a product Unsupervised neural networks can be used as a clustering technique to automatically group the customers into segments based on the similarity of their characteristic. Alternatively, supervised neural networks can be trained to learn the boundaries between customer segments based on a group of customers with known segment labels, i.e. frequent buyer, occasional buyer, rare buyer. Once market segmentation has been performed, direct marketing can be used to sell a product to customers without the need for intermediate action such as advertising or sales promotion. Customers who are contacted are already likely to respond to the products in the exhibit similar consumer behavior, as others who have responded in the past. In this way, marketers can save both time and money by avoiding contacting customers who are unlikely to respond. Bounds and Ross showed that neural networks can be used to improve response rates from the typical one to two percent, up to 95\%, simply by choosing which customers to send direct marketing mail advertisements to. Neural networks can also be used to monitor customer behavior patterns over time, and to learn to detect when a customer is about to switch to a competitor [34].

There is a marketing application which has been integrated with a neural network system. The Airline Marketing Tactician (a trademark abbreviated as AMT) is a computer system made of various intelligent technologies including expert systems. A feed forward neural network is integrated with the AMT and was trained using back propagation to assist the marketing control of airline seat allocations. The adaptive neural approach was amenable to rule expression. Additionally, the application's environment changed rapidly and constantly, which required a continuously adaptive solution. The system is used to monitor and recommend booking advice for each departure. Such information has a direct impact on the profitability of an airline and can provide a technological advantage for users of the system [35].

While it is significant that neural networks have been applied to this problem, it is also important to see that this intelligent technology can be integrated with expert systems and other approaches to make a functional system. Neural networks were used to discover the influence of undefined interactions by the various variables. While these interactions were not defined, they were used by the neural system to develop useful conclusions. It is also noteworthy to see that neural networks can influence the bottom line [36].

Neural networks change the way to use information in marketing. With such a new information technology, a company using a neural network, will eventually have affordable, near real-time access to all the raw numbers it wants. These data may be obtained from consumer credit card applications, point-of -purchase credit-card sales, and credit agency reports. The real difference among competitors will be the quality of analysis each performs and the capacity of decisions flowing from it. Neural networks help managers gather and process information, such as age, income, credit history, and products purchased [37]. 
Neural networks have been applied to a wide range of information-processing activities, such as associate memory, pattern classification and clustering, and function approximation and prediction. These applications are characterized by unstructured decision processes, multi objectives and multiple stage decision activities. Such applications may not be efficiently supported using expert and decision support systems technologies [38].

Businesses often need to forecast sales to make decisions about inventory, staffing levels, and pricing. Neural networks have had great success at sales forecasting, due to their ability to simultaneously consider multiple variables such as market demand for a product, consumers' disposable income, the size of the population, the price of the product, and the price of complementary products. Forecasting of sales in supermarkets and wholesale suppliers has been studied and the results have been shown to perform well when compared to traditional statistical techniques like regression, and human experts. The second major area where retail businesses can benefit from neural networks is in the area of market basket analyzis . Hidden amongst the daily trans action details of customers is information relating to which products are often purchased together, or the expected time delay between sales of two products [39].

Business is a diverted field with several general areas of specialization such as accounting or financial analysis. Almost any neural network application would fit into one business area or financial analysis. There is some potential for using neural networks for business purposes, including resource allocation and scheduling. There is also a strong potential for using neural networks for database mining that is, searching for patterns implicit within the explicitly stored information in databases. Most of the funded work in this area is classified as proprietary. Thus, it is not possible to report on the full extent of the work going on. Most work apply neural networks, such as the Hopfield-Tank network for optimization and scheduling [40].

Neural networks are revolutionizing virtually every aspect of financial \& investment decision-making. Financial firms worldwide are using neural networks to forecast markets, analyze credit risks, \& improve back office operations [41].

From the range of AI techniques, the one that deals best with uncertainty is the Artificial Neural Network (ANN). Dealing with One of the main areas of banking and finance that has been affected by neural networks is trading and financial forecasting. Neural networks have been applied successfully to problems like derivative securities pricing and hedging, futures price forecasting, exchange rate forecasting and stock performance and selection prediction. The success stories are numerous and have received much attention. There are many other areas of banking and finance that have been improved through the use of neural networks though. For many years, banks have used credit scoring techniques to determine which loan applicants they should lend money to. Traditionally, statistical techniques have driven the software. These days, however, neural networks are the underlying technique driving the decision making. Hecht-Nielson Co. have developed a credit scoring systems which increased profitability by $27 \%$ by learning to correctly identify good credit risks and poor credit risks . Neural networks have also been successful in learning to predict corporate bankruptcy. A recent addition to the literature on neural networks in finance is the topic of wealth creation. Neural networks have been used to model the relationships between corporate strategy short-run [42]. 
Financial health, and the performance of a company. This appears to be a promising new area of application. Financial fraud detection is another important area of neural networks in business. Visa International have an operational fraud detection systems which is based upon a neural network, and operates in five Canadian and 10 US banks. The neural network has been trained to detect fraudulent activity by comparing legitimate card use with known cases of fraud. The system saved Visa International an estimated US\$40 million within its first six months of operation alone. Neural networks have also been used in the validation of bank signatures, identifying forgeries significantly better than human experts [43].

There are many areas of operation management, particularly scheduling and planning, where neural networks have been used successfully. The scheduling of machinery, assembly lines and cellular manufacturing using neural networks have been popular research topics over the last decade. Other scheduling problems like timetabling, project scheduling and multiprocessor task scheduling have also been successfully attempted. All of these approaches are based upon the Hopfield Neural Network and the realization of Hopfield and Tank that these networks could solve complex optimization problems. Recently, alternative neural network approaches like neuro-dynamic programming have also been used to solve related problems. The other area of operations management which benefits from neural networks is quality control. Neural Networks can be integrated with traditional statistical control techniques to enhance their performance [44].

There are many areas of the insurance industry which can benefit from neural networks. Policy holders can be segmented into groups based upon their behaviors, which can help to determine effective premium pricing. Prediction of claim frequency and claim cost can also help to set premiums, as well as find an acceptable mix or portfolio of policy holders characteristics. The insurance industry, like the banking and finance sectors, is constantly aware of the need to detect fraud, and neural networks can be trained to learn to detect fraudulent claims or unusual circumstances. The final area where neural networks can be of benefit is in customer retention. Insurance is a competitive industry, and when a policy holder leaves, useful information can be determined from their history which might indicate why they have left [45].

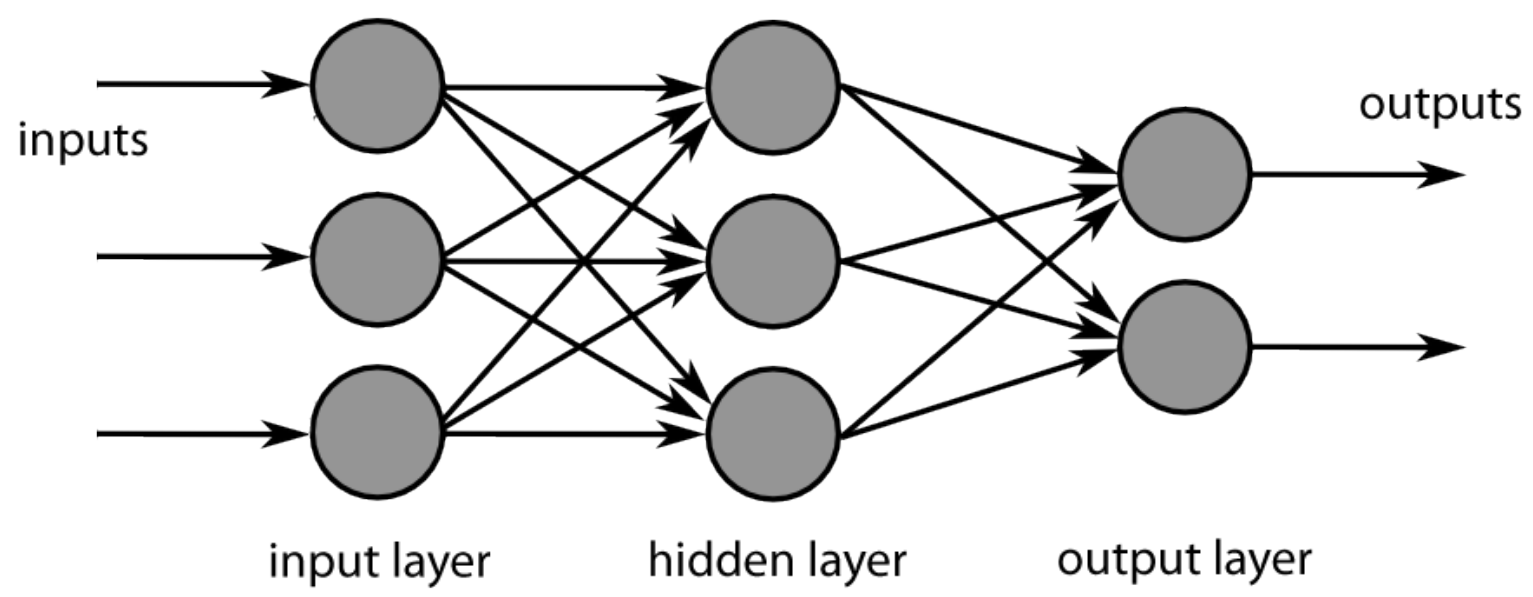

Figure 5: Neural Network 
There are also many other applications of neural networks in the telecommunications industry, and while these are more engineering applications than business applications, they are of interest to the operations researcher because they involve optimization. These include the use of neural networks to assign channels to telephone calls, for optimal network design and for the efficient routing and control of traffic [46].

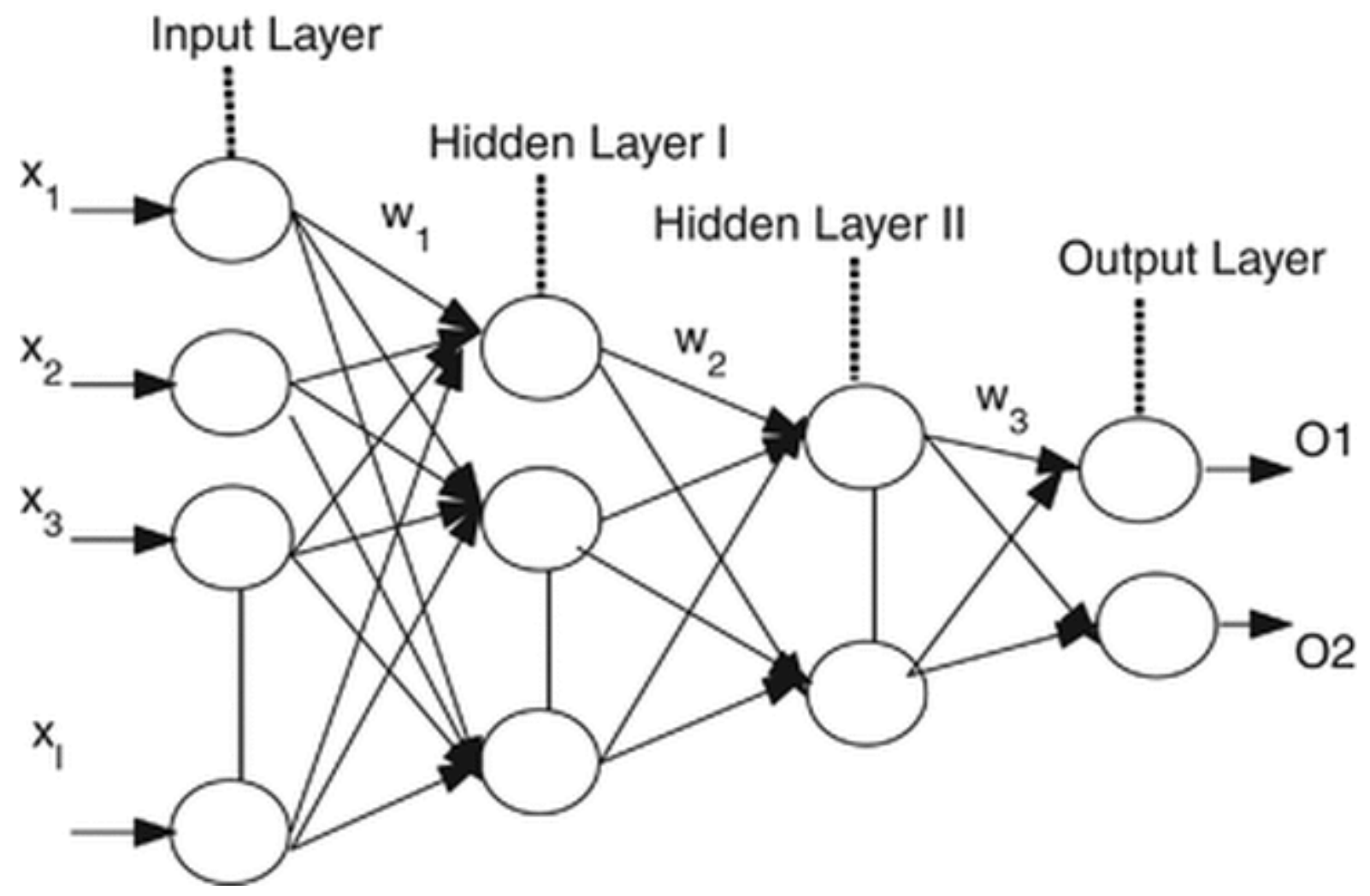

Figure 6: Forecasting Model

\section{Forecasting applications of neural networks}

The use of ANNs for forecasting has received great attention from many different fields. Given the fact that forecasting problems arise in so many different disciplines and the literature on forecasting with ANNs is scattered in so many diverse fields, it is difficult to cover all neural network forecasting applications. Table 1 provides a sample of recent business forecasting applications with ANNs reported in the literature from 1995 to 2003. As can be seen from Table 1, a wide range of business forecasting problems have been solved by neural networks. Some of these application areas include accounting (forecasting accounting earnings, earnings surprises; predicting bankruptcy and business failure), finance (forecasting stock market movement, indices, return, and risk; exchange rate; futures trading; commodity and option price; mutual fund assets and performance), marketing (forecasting consumer choice, market share, marketing category, and marketing trends), economics (forecasting business cycles, recessions, consumer expenditures, GDP growth, inflation, total industrial production, and US Treasury bond), production and operations (forecasting electricity demand, motorway traffic, inventory, new product development project success, IT project escalation, product demand or sales, and retail sales), international business (predicting joint venture performance, foreign exchange rate), real estate (forecasting residential construction demand, housing value), tourism and transportation (forecasting 
tourist, motorway traffic, and international airline passenger volume), and environmental related business (forecasting ozone level and concentration, air quality) [47].

\section{Conclusion}

Artificial neural networks have emerged as an important tool for business forecasting. ANNs have many desired features that are quite suitable for practical forecasting applications. This chapter provides a general overview of the neural networks for forecasting applications. Successful forecasting application areas of ANNs, as well as critical modeling issues are reviewed. It should be emphasized that each forecasting situation requires a careful study of the problem characteristics, prudent design of modeling strategy, and full consideration of modeling issues. Many rules of thumb in ANNs may not be useful for a new application, although good forecasting principles and established guidelines should be followed. ANNs have achieved remarkable successes in the field of business forecasting. It is, however, important to note that they may not be a panacea for every forecasting task under all circumstances. Forecasting competitions suggest that no single method, including neural networks, is universally the best for all types of problems in every situation. Thus, it may be beneficial to combine several different models in improving forecasting performance. Indeed, efforts to find better ways to use ANNs for forecasting should never cease. The subsequent chapters of this book will provide a number of new forecasting applications and address some practical issues in improving ANN forecasting performance.

\section{References}

[1] Armstrong, J.S., Long-Range Forecasting, Second Edition, New York: Wiley, 1985.

[2] Bell, T., G. Ribar, and J. Verchio, "Neural Nets vs. Logistic Regression," presented at the University of Southern California Expert Systems Symposium, November, 1989.

[3] Chatfield, C., "Neural Networks: Forecasting Breakthrough or Passing Fad?," International Journal of Forecasting, 1993, 9, 1-3.

[4] Collopy, F. and J.S. Armstrong, "Expert Opinions about Extrapolation and the Mystery of the Overlooked Discontinuities," International Journal of Forecasting, 1992, 8, 575-582.

[5] Connor, D., "Data Transformation Explains the Basics of Neural Networks," EDN, May 12, 1988, 138-144.

[6] Cybenko, G., "Continuous Valued Neural Networks with Two Hidden Layers Are Sufficient," Technical Report, Department of Computer Science, Tufts University, 1988.

[7] Dalrymple, D.J., "Sales Forecasting Practices: Results of a United States Survey," International Journal of Forecasting, 1987, 3, 379-392.

[8] De Gooijer, I.G. and K. Kumar, "Some Recent Developments in NonLinear Modelling, Testing, and Forecasting," International Journal of Forecasting, 1992, 8, 135-156. 
[9] Donaldson, R.G., M. Kamstra, and H.Y. Kim, "Evaluating Alternative Models for Conditional Stock Volatility: Evidence from International Data," Working Paper, University of British Columbia, 1993.

[10] Duliba, K., "Contrasting Neural Nets with Regression in Predicting Performance," Proceedings of the 24th Hawaii International Conference on System Sciences, 1991, Vol. 4, 163-170.

[11] Dutta, S. and S. Shekhar, "Bond Rating: A Non-Conservative Application of Neural Networks," Proceedings of the 1988 International Conference on Neural Networks, 1988, Vol. 2, 443450.

[12] Foster, B., F. Collopy, and L. Ungar, "Neural Network Forecasting of Short, Noisy Time Series," presented at the ORSA TIMS National Meeting, May, 1991.

[13] Gluck, M.A. and G.H. Bower, "Evaluating an Adaptive Model of Human Learning," Journal of Memory and Language, 1989, 27, 166-195.

[14] Gorr, W.I., D. Nagin, and J. Szczypula, "Comparative Study of Artificial Neural Networks and Statistical Methods for Predicting Student Grade Point Averages, " Working Paper, Carnegie Mellon University, 1993.

[15] Granger, R., J. Ambros-Ingerson, U. Staubli, and G. Lynch, "Memorial Operation of Multiple, Interacting Simulated Brain Structures," in Neuroscience and Connectionist Models, M. Gluck and D. Rumelhart, eds., Hillsdale: Erlbaum Associates, 1989.

[16] Hiew, M. and G. Green, "Beyond Statistics. A Forecasting System That Learns," The Forum, 1992, Vol. 5, pp. 1 and 6.

[17] Hill, T., M. O'Connor, and W. Remus, "Neural Networks for Time Series Forecasting," Working Paper, University of Hawaii, 1993, Third round at Management Science.

[18] Hill, T. and W. Remus, "Neural Network Models for Intelligent Support of Managerial Decision Making," Decision Support Systems, 1993, Forthcoming.

[19] Hoptroff, R.G., "The Principles and Practice of Time Series Forecasting and Business Modeling Using Neural Nets," Neural Computing \& Applications, 1993, 1, 59-66.

[20] Hornik, K., M. Stinchcombe, and H. White, "Multilayer Feedforward Networks are Universal Approximators," Neural Networks, 1989, 2(5), 359-366.

[21] Kang, S., An Investigation of the Use of Feedforward Neural Networks for Forecasting, Ph.D. Dissertation, Kent State, 1991.

[22] Koster, A., N. Sondak, and W. Bourbia, "A Business Application of Artificial Neural Network Systems," The Journal of Computer Information Systems, 1990, Vol. XI, 3-10. 
[23] Lawrence, M.J., R.H. Edmundson, and M.J. O'Connor, "An Examination of the Accuracy of Judgemental Extrapolation of Time Series," International Journal of Forecasting, 1985, 1, 25-35.

[24] Makridakis, S., A. Anderson, R. Carbone, R. Fildes, M. Hibon, R. Lewandowski, J. Newton, E. Parzen, and R. Winkler, "The Accuracy of Extrapolation (Time Series) Methods: Results of a Forecasting Competition," Journal of Forecasting, 1982, 1, 111-153.

[25] Makridakis, S. and R. L. Winkler, "Averages of Forecasts: Some Empirical Results," Management Science, 1985, 29, 987-996.

[26] Marquez, L., Function Approximation Using Neural Networks: A Simulation Study, Ph.D. Dissertation, University of Hawaii, 1992.

[27] Marquez, L., T. Hill, W. Remus, and R. Worthley, "Neural Network Models as an Alternative to Regression," in Neural Network Applications in Finance and Investing edited by R. Trippi and E. Turban, Chicago: Probus Publishing, 1992, 435-450.

[28] Nelder, J. and R. Mead, "The Downhill Simplex Method," Computer Journal, 1965, 7, 308-310.

[29] Odom, M. and R. Sharda, "A Neural Network Model for Bankruptcy Prediction," Proceedings of the 1990 International Joint Conference on Neural Networks, 1990, Vol. 2, 163-168.

[30] Pack, D.J. and D.J. Downing, "Why Didn't Box-Jenkins Win (Again)?" presented at the Third International Symposium on Forecasting, 1983.

[41] Raghupathi, W., L. Schkade, and R. Bapi, "A Neural Network Application for Bankruptcy Prediction," Proceedings of the 24th Hawaii International Conference on System Sciences, 1991, Vol. 4, 147-155.

[32] Remus, W., "A Study of the Impact of Graphical and Tabular Displays and Their Interaction with Environmental Complexity," Management Science, 1987, Vol. 33, 1200-1205.

[33] Remus, W. and T. Hill, "Neural Network Models of Managerial Judgment," Proceedings of the 23rd Hawaii International Conference on System Sciences, 1990, Vol. 4, 340-344. Forthcoming in Advances in Artificial Intelligence in Business and Finance.

[34] Roy, J. and J. Cosset, "Forecasting Country Risk Ratings Using a Neural Network," Proceedings of the 23rd Hawaii International Conference on System Sciences, 1990, Vol. 4, 327-334.

[35] Rumelhart, D. and J. McClelland, Parallel Distributed Processing, Cambridge: MIT Press, 1986.

[36] Rumelhart, D., G.E. Hinton, and R.J. Williams, "Learning Representations by Back-Propagating Errors," Nature, 1986, 323, 533-536. 
[37] Sharda, R. and R. Patil, "Neural Networks as Forecasting Experts: An Empirical Test," Proceedings of the 1990 International Joint Conference on Neural Networks Meeting, 1990, Vol.

[38] Sharda, R. and R. Patil, "Connectionist Approach to Time Series Prediction: An Empirical Test," Journal of Intelligent Manufacturing, 1992, Forthcoming.

[39] Sietsma, J. and R. Dow, "Creating Artificial Neural Networks That Generalize," Neural Networks, 1991, 4, 67-79.

[40] Surkan, A. and J. Singleton, "Neural Networks for Bond Rating Improved by Multiple Hidden Layers," Proceedings of the 1990 International Joint Conference on Neural Networks, 1990, Vol. 2, 157-162.

[41] Tam, K.Y., "Neural Network Models and the Prediction of Bank Bankruptcy," Omega, The International Journal of Management Science, 1991, 19, 429-445.

[42] Tam, K.Y. and M.Y. Kiang, "Managerial Applications of Neural Networks: The Case of Bank Failure Predictions," Management Science, 1992, 38, 926-947.

[43] Tang, Z., C. de Almeida, and P. Fishwick, "Time Series Forecasting Using Neural Networks vs. BoxJenkins Methodology," Simulation, 1991, Vol. 57, 303-310.

[44] Wasserman, P.D., Neural Computing: Theory and Practice, Van Nostrand Reinhold: New York, 1989.

[45] Weigend, A., B. Huberman, and D. Rumelhart, "Predicting the Future: A Connectionist Approach," International Journal of Neural Systems, 1990, 1, 193-209.

[46] Widrow, B. and S.D. Sterns, Adaptive Signal Processing, Englewood Cliffs, NJ: Prentice-Hall, 1985.

[47] Yoon, Y. and G. Swales, "Predicting Stock Price Performance," Proceedings of the 24th Hawaii International Conference on System Sciences, 1991, Vol. 4, 156-162. 